\title{
CORRIGENDUM
}

\section{An introduction to Bayesian inference in gravitational-wave astronomy: parameter estimation, model selection, and hierarchical models-Corrigendum}

\author{
Eric Thrane ${ }^{1,2}$ and Colm Talbot ${ }^{1,2}$ \\ ${ }^{1}$ Centre for Astrophysics, School of Physics and Astronomy, Monash University, VIC 3800, Australia and ${ }^{2}$ OzGrav: The ARC Centre of Excellence for Gravitational-Wave \\ Discovery, Clayton, VIC 3800, Australia
}

doi: 10.1017/pasa.2019.2, published by Cambridge University Press, March 11, 2019.

We correct two errors in Thrane and Talbot (2019).

1. In the original version of this article, we included a subsection in Appendix E, "Selection effects with a single event." This section included formulas with errors including Eq. 89 and Eq. 95 of the arxiv version (Eq. E2 and Eq. E8 in the version published in PASA). Moreover, the section included a conceptual error since the idea of selection effects for single events does not make sense. Selection effects are intrinsically related to population studies, so they simply do not affect the analysis of single detections. It is interesting to consider how this comes about mathematically. While the single-event det likelihood gains a factor of $p_{\operatorname{det}}^{-1}$ (as correctly noted in the original article), the single-event det prior picks up a compensating factor of $p_{\text {det }}$, because the prior for detected events is not the same as the original (no det) prior. Since the det posterior is proportional to the product of the likelihood and the prior, these two factors cancel, giving the original (no det) likelihood. A revised version of the appendix is presented below.

2. In eight places we referred to "the odds ratio." However, we should have referred simply to "the odds." In statistics, the odds refers to a ratio of probabilities. When we multiply the Bayes factor by the prior odds, we obtain the posterior odds. The odds ratio, which is also a statistical term, refers to a ratio of ratios.

The following is the revised version of Appendix E.

\section{Appendix E. Selection Effects}

In this section, we discuss how to carry out inference while taking into account selection effects, which arise from the fact that some events are easier to detect than others. We loosely follow the arguments from Abbott et al. (2016); however, see also Mandel et al. (2018); Fishbach et al. (2018).

Author for correspondence: Eric Thrane, E-mail: eric.thrane@monash.edu Cite this article: Thrane E and Talbot C. (2020) An introduction to Bayesian inference in gravitational-wave astronomy: parameter estimation, model selection, and hierarchical models-Corrigendum. Publications of the Astronomical Society of Australia 37, e36, 1-2. https://doi.org/10.1017/pasa.2020.23
Some gravitational-wave events are easier to detect than others. All else equal, it is easier to detect binaries if they are closer, higher mass (at least, up until the point that they start to go out of the observing band), and with face-on/off inclination angles. More subtle selection effects arise due to black hole spin (see, e.g., $\mathrm{Ng}$ et al., 2018). Typically, a gravitational-wave event is said to have been detected if it is observed with a matched-filter signal-to-noise ratio-maximized over extrinsic parameters $\theta_{\text {extrinsic }}-$ above some threshold $\rho_{\text {th }}$

$$
\rho_{\mathrm{mf}}^{\prime} \equiv \max _{\theta_{\text {extrinsic }}}\left(\rho_{\mathrm{mf}}\right)>\rho_{\mathrm{th}} .
$$

Usually, $\rho_{\text {th }}=8$ for a single detector or $\rho_{\text {th }}=12$ for a $\geq 2$ detector network.

Selection effects are characterised by $p_{\text {det }}$, the probability that a signal exceeds the detection threshold. There are different ways to calculate $p_{\text {det }}$ in practice. The probability density function for $\rho_{\mathrm{mf}}$ given $\theta$-the distribution of $\rho_{\mathrm{mf}}$ arising from random noise fluctuations-is a normal distribution with mean $\rho_{\text {opt }}$ and unit variance

$$
p\left(\rho_{\mathrm{mf}}^{\prime} \mid \theta\right)=\frac{1}{2 \pi} \exp \left(-\frac{1}{2}\left(\rho_{\mathrm{mf}}^{\prime}-\rho_{\mathrm{opt}}(\theta)\right)^{2}\right),
$$

see Fig. 1. Thus,

$$
\begin{aligned}
p_{\text {det }}(\theta) & =\int_{\rho_{\text {th }}}^{\infty} d x \frac{1}{\sqrt{2 \pi}} \exp \left(-\frac{1}{2}\left(x-\rho_{\text {opt }}(\theta)\right)^{2}\right) \\
& =\frac{1}{2} \operatorname{erfc}\left(\frac{\rho_{\text {th }}-\rho_{\text {opt }}(\theta)}{\sqrt{2}}\right) .
\end{aligned}
$$

Alternatively, one may express $p_{\text {det }}$ as the ratio of the "visible volume" $\mathcal{V}(\theta)$ to the total spacetime volume $\mathcal{V}_{\text {tot }}$

$$
p_{\text {det }}(\theta)=\frac{\mathcal{V}(\theta)}{\mathcal{V}_{\text {tot }}} .
$$

The visible volume is typically calculated numerically with injected signals.

Given a population of $N$ events,

$$
\mathcal{L}(d, N \mid \Lambda, \operatorname{det})=\frac{1}{p_{\operatorname{det}}(\Lambda \mid N)} \mathcal{L}(d, N \mid \Lambda, R) .
$$

In analogy to Eq. 5 , the $p_{\text {det }}$ normalization factor can be calculated using the visible volume as a function of the hyper-parameters $\Lambda$ 


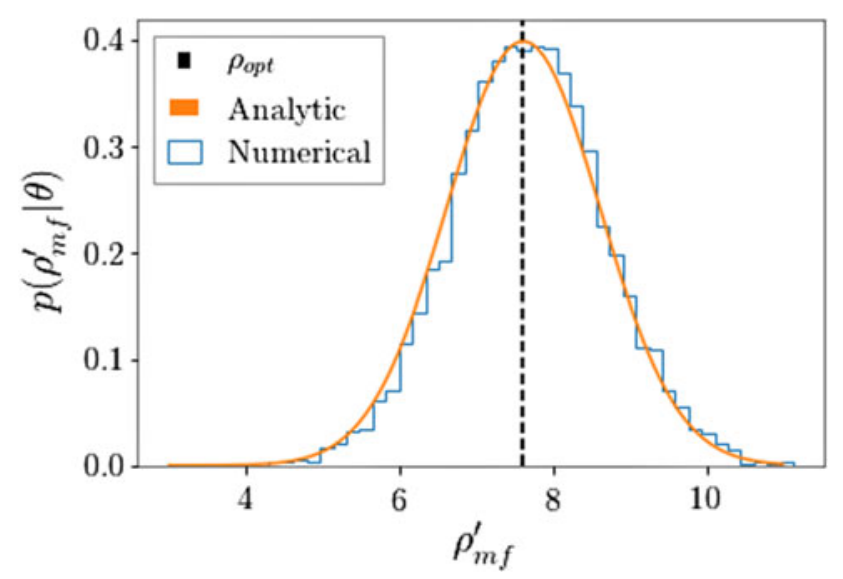

Figure 1. The distribution of matched filter signal-to-noise ratio maximized over phase for the same template in many noise realisations (blue). The distribution peaks at $\rho_{\text {opt }}=7.6$ (dashed black). The theoretical distribution (Eq. 2 ) is shown in orange.

$$
\mathcal{V}(\Lambda) \equiv \int d \theta \mathcal{V}(\Lambda) \pi(\theta \mid \Lambda)
$$

Naively, one might expect that

$$
p_{\text {det }}(\Lambda \mid N)=\left(\frac{\mathcal{V}(\Lambda)}{\mathcal{V}_{\text {tot }}}\right)^{N},
$$

but this expression is incorrect because it does not marginalize over the Poisson-distributed rate, which ends up changing the answer. Marginalizing over the rate, we obtain

$$
\begin{aligned}
p_{\text {det }}(\Lambda \mid N) & =\int d R\left(\frac{\mathcal{V}(\Lambda)}{\mathcal{V}_{\text {tot }}}\right)^{N} \pi(N \mid R) \pi(R) \\
& =\int d R\left(\frac{\mathcal{V}(\Lambda)}{\mathcal{V}_{\text {tot }}}\right)^{N}\left[e^{-R \mathcal{V}(\Lambda)} \frac{\mathcal{V}(\Lambda)^{N} R^{N}}{N !}\right] \pi(R) \\
& =\left(\frac{\mathcal{V}(\Lambda)}{\mathcal{V}_{\text {tot }}}\right)^{N}\left[\int d R e^{-R \mathcal{V}(\Lambda)} \frac{\mathcal{V}(\Lambda)^{N} R^{N}}{N !}\right] \pi(R) .
\end{aligned}
$$

Note that $p_{\text {det }}$ depends on our prior for the rate $R$. If we choose a uniform-in-log prior $\pi(R) \propto 1 / R$, we obtain

$$
p_{\text {det }}(\Lambda \mid N) \propto\left(\frac{\mathcal{V}(\Lambda)}{\mathcal{V}_{\text {tot }}}\right)^{N},
$$

which reproduces the results from Abbott et al. (2018). Note that

$$
\mathcal{L}(d \mid \Lambda, \operatorname{det}) \neq \int d \theta \mathcal{L}(d \mid \theta, \operatorname{det}) \pi(\theta \mid \Lambda)
$$

\section{References}

Abbott B. P., et al., 2016, Phys. Rev. X, 6, 041015

Abbott B. P., et al., 2018, Binary Black Hole Population Properties Inferred from the First and Second Observing Runs of Advanced LIGO and Advanced Virgo, https://arxiv.org/abs/1811.12940

Fishbach M., Holz D. E., \& Farr W. M., 2018, Astrophys. J. Lett., 863, L41

Mandel I., Farr W. M., Gair J. R., 2018, Extracting distribution parameters from multiple uncertain observations with selection biases, https://arxiv.org/abs/1809.02063

Ng K. K. Y., Vitale S., Zimmerman A., Chatziioannou K., Gerosa D., Haster C.-J., 2018, Phys. Rev. D, 98, 083007

Thrane E., \& Talbot C., 2019, PASA, 36, e010 